\title{
Stay Tuned! \\ An Automatic RSS feeds Trans-coder
}

\author{
Patrick Salamin, Alexandre Wetzel, Daniel Thalmann, and Frédéric Vexo \\ VRLab - EPFL, Switzerland \\ patrick.salamin@epfl.ch, \\ WWW home page: http://vrlab.epfl.ch
}

\begin{abstract}
News aggregators are widely used to read RSS feeds but they require the user to be in front of a screen. While moving, people usually do not have any display, or very small ones. Moreover, they need to perform actions to get access to the news: download a tool, choose to generate audio files from the news, and send them to e.g. an MP3 player. We propose in this paper a system that automatically detects when the user leaves the computer room and directly sends the trans-coded news onto the user Smartphone. All the aggregated news are then transmitted to the user who can listen to them without any action. We present in this paper such a system and the very promising results we obtained after testing it.
\end{abstract}

Key words: User context awareness, RSS reader, Trans-coding, Geolocalization

\section{Introduction}

Many people are using news aggregators but sometimes (e.g. while moving) they do not have at their disposal a screen, or a too small one to read the RSS feeds. They must then perform conscious actions to have access to these RSS feeds. A solution is to download one of the available-on-the-Internet tools. But in this case, the user must press a button to generate the audio files, connect his/her MP3 player to the computer, and accomplish the file transfer with a "drag and drop" operation onto the player.

We propose in this paper a solution executing an automatic transfer of the trans-coded RSS feeds onto the Smartphone that will directly continue to read the news. Being aware of the user context (the user location) and able to transcode the textual news onto audio files, our system automatically decides when it must send the files to the user Smartphone.

We will first present an overview of the current technologies and researches on the fields of context awareness and trans-coding processes. After this, we will describe more in detail our concept and the needed system architecture. We will finally conclude with a discussion on the results of the previously described tests we made, but also on the further improvements of our application. 


\section{Related works}

As the user cannot read the news while walking, we need to trans-code the information. Some very promising researches were done within the trans-coding domain, especially with the auditory and visual modalities. Jacquemin et al. e.g. developed a system involving audio- and visuo-rendering to mimic gusts of wind blowing a veil [1]. We also developed in 2007 an application allowing blind people to recognize 3D digital content with the help of a PDA [2]. By the way, there already exist several trans-coders to podcast; we could cite for instance Netvibes $^{1}$, Feedburner ${ }^{2}$, Google reader ${ }^{3}$, etc. But the main disadvantage of these tools resides in the manual operations needed to use them.

With the apparition of the "user context awareness" concept, the system must react according to the user context instead of requiring an interaction with the user like pushing a button. In our case, the system should be able to locate the user. An invisible indoor geo-localization system could lead to a fully automated process and a real follow of the user [3]. The system, aware of the user context, must then be able to detect the user displacements, or at least the user location, in order to switch from the screen to another way to transmit the information. The mostly used system for geo-localization is the Global Positioning System (GPS), but it only works outdoor and our experiment takes place indoor. There exist several other technologies using specific hardware like infrared, magnetic, ultrasound, etc. But even if they are very precise, they are also very expensive and usually limited to one room. More recent systems tend to use cheaper and already deployed technologies like GSM, Wi-Fi, or Bluetooth. For example, RADAR [4][5] is a tracking system working with the signal strength of radio-based devices. This system inspired several researchers who tried to improve different aspects of the system: accuracy [6][7], localization in high signal fluctuation areas [8], and only using access points [9][10]. But in our experiment we only need to know if the user left his/her computer and the researches of Dhawan [11], [12], and [13] also inform us on range and possible co-existence of Bluetooth and Wi-Fi.

Based on the researches cited above, we decided to trans-code RSS feeds into audio files that will be read on a Smartphone. The user's proximity detection will be performed with the help of the Bluetooth and the data will be transmitted over IP with a previously installed Wi-Fi network. Our solution is described more in detail in the following sections.

\section{Concept description}

Nowadays, most of people with a computer use news aggregators like Google News. But, when people are no more in front of their computer, they still should

\footnotetext{
${ }^{1}$ http://www.netvibes.com/

${ }^{2}$ http://www.feedburner.com/

${ }^{3}$ http://www.google.com/reader/
} 
be able to get access to the news they have aggregated. They have then to find a way to trans-code the RSS feeds and to send them to a player they will wear while moving.

As shown in the previous section, there already exist tools that translate news into podcast. Such systems require the user to decide which news must be translated and when. Moreover, once trans-coded, the user must copy them onto an audio player he/she will wear while walking, driving, etc.

Our system performs all these actions automatically (Figure 1). It first stores the news aggregated by the user and automatically trans-codes them into audio files. Then, it detects when the user leaves the computer room and sends to the Smartphone all the unread aggregated news to allow the user to listen to them. Finally, it updates the content depending on the possible newly added RSS feeds in the reader list. The only one data entered by the user is the Smartphone IP address on the Wi-Fi network.

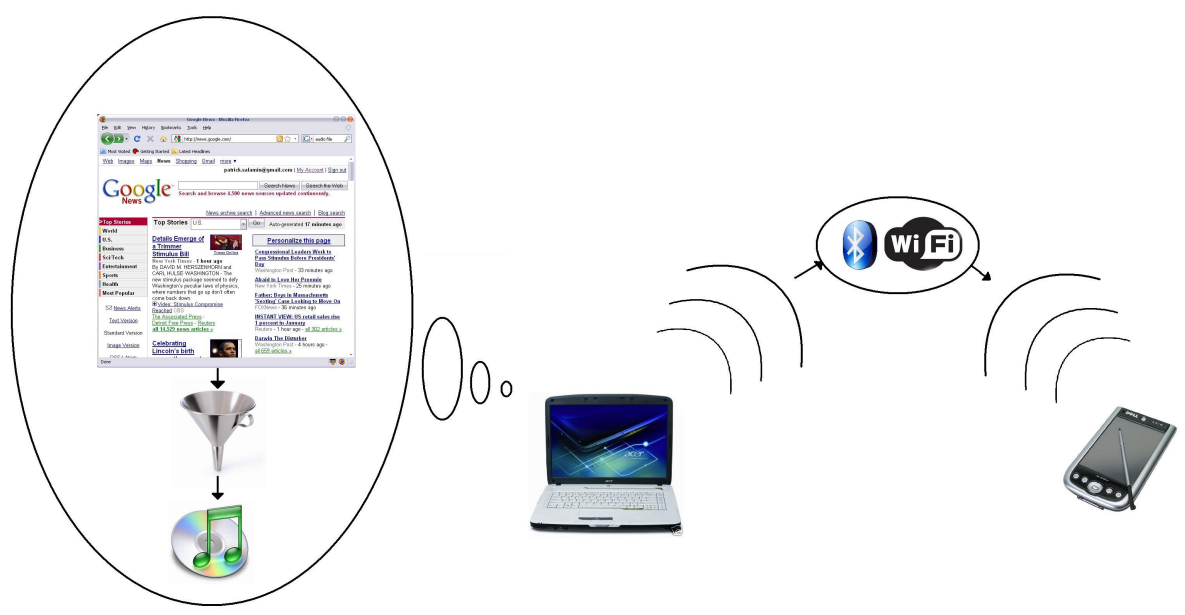

Fig. 1. Schema of our concept: from RSS feeds on the computer (from text into audio files) to an audio player on the smartphone

\section{System architecture}

In this section, we present more in detail, the previously described steps of our application and the required equipment.

\subsection{Software}

We present in this part the different steps of our application. We decided to program the complete application in JAVA in order to avoid restrictions due to the operating system. 
The first step concerns the news trans-coding (left side of Figure 1). We use a free text-to-speech (TTS) library that takes a string as input and create an audio file. Obviously, news language must be precised (imagine an English text read with French rules). Most of the time, the language is precised as a tag in the RSS feed but there are many variations, for instance "en", "us", "uk", and all possible combinations of them may happen to indicate an English feed. Once trans-coding operation done, we get a readable audio file ready to be sent to the user's Smartphone.

At every moment our system is "aware of the user context". In our case, user context awareness concerns his/her location, and especially the distance between him/her and the computer screen. As previously mentioned in the related works section, there exist several ways to detect the location of someone but, for practical reasons, we decided to use Bluetooth detection. There are two advantages of working with Bluetooth. First, Bluetooth-equipped material are only detected when they are in a range of few meters from the Bluetooth dongle linked to the computer. Secondly, almost every Smartphone is fitted out Bluetooth.

Once our system detects that the user is leaving, it sends the audio files to the Smartphone. We use Wi-Fi connection to perform this task for two reasons. As the Wi-Fi range is larger than the Bluetooth one, there is still few seconds to send the audio files to the Smartphone after the user's leaving is detected. Secondly, almost every Smartphone is also equipped with a Bluetooth antenna. After this, the application running in background on the Smartphone, a audio file player starts to read the news and the user can listen to them while walking in the street.

\subsection{Hardware}

Our system, a client-server application, is composed of two "entities": the master computer (server) and the slave Smartphone (client). Both are connected to a $\mathrm{Wi}-\mathrm{Fi}$ router and Bluetooth-paired. As mentioned above, Bluetooth range ${ }^{4}$ is smaller than Wi-Fi range ${ }^{5}$, which allows to send data from the computer to the Smartphone via Wi-Fi directly after Bluetooth disconnects.

During our experiments, on the server side, we use a Dell Inspiron $640 \mathrm{~m}$ for the desktop station. It is an Intel Core 2 Duo Processor T5600 (1.83GHz) and it is equipped with Wi-Fi and Bluetooth antennas (Intel PRO WLAN 3945 Wireless card, respectively Dell True Mobile 350 Bluetooth card). Its installed operating system is Windows XP SP3 and we worked with the platform JAVA SE 6 . There is indeed no need of a powerful computer to simply trans-code small text files to audio ones and send them via IP.

The client is a Dell Axim 50v with a 3.7in VGA display providing a software resolution of $640 \times 480$ pixels. It is composed of a $624 \mathrm{MHz}$ Intel Xscale PXA270 processor, a 128MB Flash ROM and a 64MB SDRAM and is equipped with a

\footnotetext{
${ }^{4}$ http://en.wikipedia.org/wiki/Bluetooth

${ }^{5}$ http://en.wikipedia.org/wiki/IEEE_802.11b-1999
} 
802.11b Wi-Fi and Bluetooth antennas. Its installed operating system is Windows Mobile 5 and we used the EVE Virtual Machine 1.27 to program our application in Java.

The first advantage of our system is to be completely automatic. Moreover, our system is to be very low-cost. Indeed, Smartphone become more and more common and the used transmission protocol are also very standard. This system could then be used by almost anyone at home. Some everyday scenarios are presented and analyzed in the following section to validate our system.

\section{The experiment}

In this section, we first present the participants who take part to the experiment. We then describe the protocol followed during the experiment and their analysis in the following section.

\subsection{Participants}

Twelve people from 19 to 46 (mean: 26 years old) years old voluntarily took part to the experiment. Most of them (10) already read RSS feeds on the Internet and all of them had already used a Smartphone.

\subsection{Protocol}

Before we start the experiment, we give to the users an overview (or reminder) of RSS feeds, the used material, and the purpose of the system. We then explain to them briefly the scenario of the experiment and which actions they will be asked to perform. Finally, we give them a Smartphone and show them the computer (server) on which they will start the experiment. At this moment, both applications (on the client and on the server) are already started. Notice also that both (computer and Smartphone) are already Bluetooth-coupled and that we already entered the client IP into the server application.

Once the user is informed, we invite him/her to seat in front the computer screen (server) to aggregate some news and start to read them. After few minutes, we ask them to stand up and to leave the room with the Smartphone (Figure 2). The final step consists in listening the sent news and then to come back to the computer, which lead our system to make an update concerning the status of the pieces of news (to consider them as read or not).

All the three steps cited above are performed at least two times. The first loop is for the adaption to the system (we sometimes needed to coach the user). During the following loop(s), we only are observers while the user performs the tasks of the experiment. The experimentation usually lasts around fifteen minutes. 

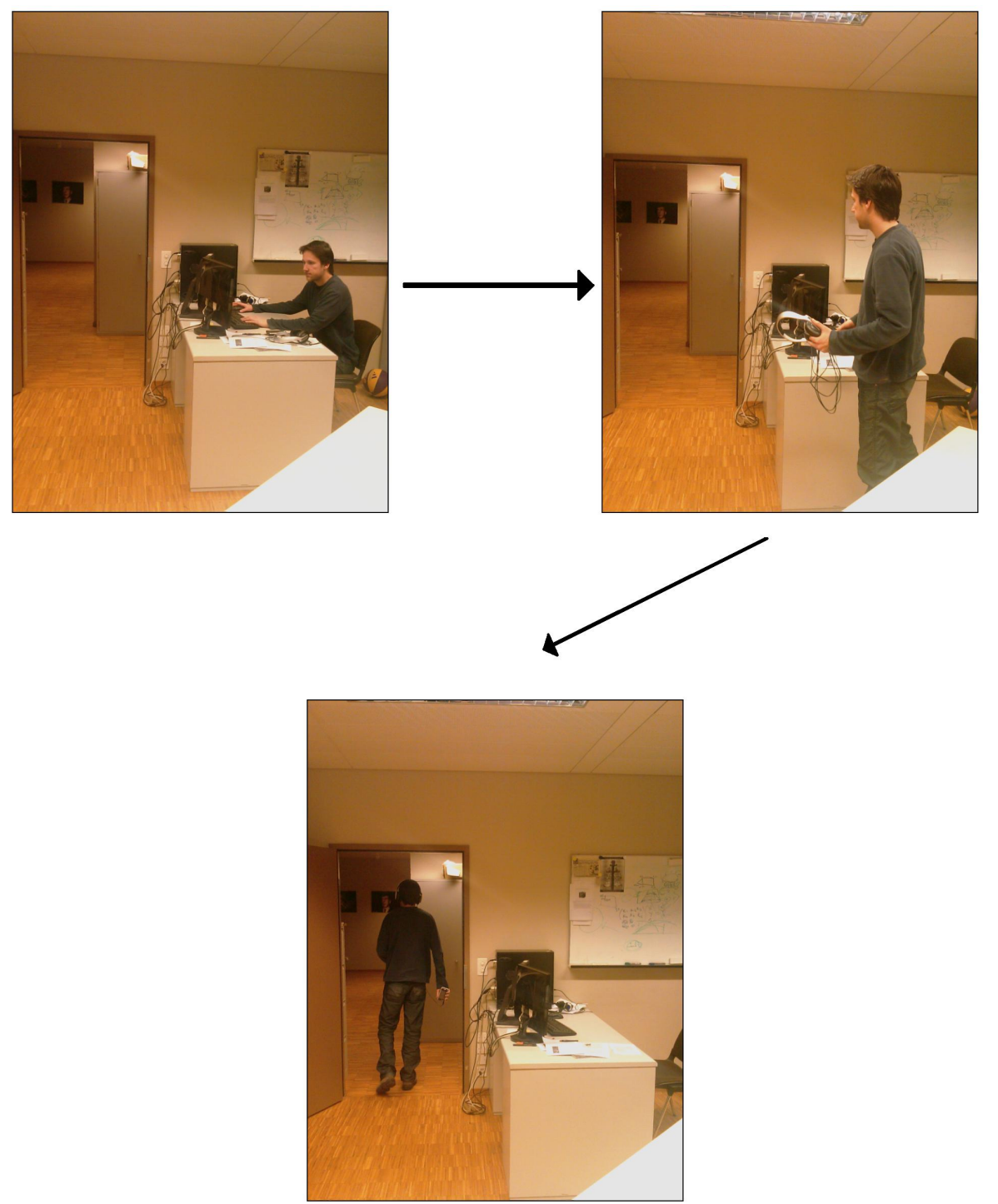

Fig. 2. Scenario of the experiment: the user first stays in front of the computer to read the news; He/she leaves the desktop station to go working; He/she continues to listen to the news while walking in the street. 


\subsection{Questionnaire}

Once the experiment performed, we proposed a SUMI-like (Software Usability Measurement Inventory) questionnaire ${ }^{6}[14]$ to the users. Indeed, several types of validity studies [15][16][17] have already been conducted with SUMI, whose one of them concerns laboratory-based studies (carried out in the Human Factors Research Group). This questionnaire is composed of two parts that we describe here.

The first part is composed of questions about the user profile like the age, gender, but also if the user is used to work with a computer and a Smartphone. This part concludes with questions about the training for using the system (availability and length) and if the time to use the system was also adequate.

The second part of the questionnaire is composed of fifty statements. The user must answer to all of them by marking one of the three proposed boxes labeled: "Disagree", "Undecided", and "Agree". It is also firstly noticed that marking the "Undecided" boxes means that the user cannot make up his/her mind, or that the statement has no relevance to the software or the situation. Secondly, it is added that marking the "Disagree" or "agree" boxes does not necessarily indicate a strong disagreement (respectively agreement) but only a general feeling most of the time.

The questions of this second part concern various topics: responsiveness of the software, quality of proposed instructions, global satisfaction about the software, possible improvements, intuitiveness, and attractiveness of the software. In the next section, we will analyze the users' answers and their behavior during the experiment.

\section{Results}

Globally, most of the users enjoyed the system. Every step was performed by every user, even if some of them needed more time to adapt to the system. We will now first present the users' behavior during the experimentation and then the answers they give to our questionnaire.

\subsection{Behavioral analysis}

The first step was well-performed by every user at the second loop. Indeed, during the first loop (also called adaptation loop), few of them were discovering the RSS feeds aggregators, so they needed a bit more time to understand the concept. Anyway, using an RSS aggregator seems to be a very common (or at least easy) action to perform.

At each loop of the experiment, we ask the user to stand up and to leave the computer room with the Smartphone. After being out of the room, most of users stay in the corridor during the first loop. They are hesitating about what

\footnotetext{
${ }^{6}$ http://sumi.ucc.ie/
} 
they have to do, and especially if they can go further or must stay. In this case, we explain them that they can go as if they were going to work or if they had anything else to do. Notice also that most of them were also surprised when the Smartphone started to play the audio files.

This hesitation and surprise do not appear at the second loop and every user goes further, listening to the news. It is also important to notice that a problem appeared with two people during this last step. As they really hurry to stand up and almost rush out of the room, our system did not have enough time to send the data to the Smartphone. Indeed, as our system detects the user's disappearing, the Smartphone was already out of the Wi-Fi range and could not receive the server data. Except these two cases, every experiment went fine. Two users even asked us if there exist a similar application for emails reading was available.

\subsection{Questionnaire trends}

Our adapted SUMI questionnaire was filled by every participant. Its first part, concerning the users' profile, reveals us that one experiment loop for training was widely enough for all the participants. Globally, after two loops, the users feel comfortable with the application and almost all of them would like to use it in the day life. Finally, number of loops in the experiment is also considered as adequate.

The questions of the second part reveal us that our software is very accurate and fast enough for everyone but two. Indeed, the Bluetooth detection seems not to be fast enough for people rushing out of the room because they are very late. Notice also that the update action (to add new RSS feeds and delete already read ones) was considered as "a bit too long" by eight people. Finally, they seem to be a bit surprised at the beginning with the Smartphone that automatically starts to read news but after the first loop, they really enjoyed our application.

Our system was then considered as very attractive, easy to use, and intuitive enough, even if improvements can be done in the future.

\section{Conclusion and further works}

The obtained results confirm our hypotheses. Being able to have access to one's aggregate news all the time (and not only in front of a computer screen) is a very attractive concept.

It seems also that our application reached the planned objectives: attractiveness, intuitiveness, and efficiency. Because of the users' enthusiasm, we may deduce that our application is attractive. As mentioned above, some users even asked us if there already exist a similar version of our application to read emails. In addition, most of the users did neither need any help nor too much time to feel comfortable with our application. Finally, the users really appreciate the concept of the "following trans-coded news". 
Nevertheless, some users deplored the "update" and "sending information" steps speed. These steps are indeed the bottlenecks of our application. In order to solve this problem, a first solution would be to send the RSS feeds as text files to the Smartphone and trans-code them after. But this would not solve the problem of the two very fast users. Another solution would be to send data via $3 \mathrm{G}^{7}$ instead of Wi-Fi. In this case, data transmission would be slower but would no more suffer from "out of range" user.

\section{Acknowledgments}

This research has been partially supported by the European Coordination Action: FOCUS K3D (http://www.focusk3d.eu).

\section{References}

1. Christian Jacquemin and Serge de Laubier. Transmodal feedback as a new perspective for audio-visual effects. In NIME '06: Proceedings of the 2006 conference on New interfaces for musical expression, pages 156-161, Paris, France, France, 2006. IRCAM - Centre Pompidou.

2. Patrick Salamin, Daniel Thalmann, and Frederic Vexo. Visualization Learning for Visually Impaired People. In The 2nd International Conference of E-Learning and Games: Edutaiment 2007, pages 171-181, 2007.

3. Gregory D. Abowd, Anind K. Dey, Peter J. Brown, Nigel Davies, Mark Smith, and Pete Steggles. Towards a better understanding of context and context-awareness. In HUC '99: Proceedings of the 1st international symposium on Handheld and Ubiquitous Computing, pages 304-307, London, UK, 1999. Springer-Verlag.

4. P. Bahl and V. N. Padmanabhan. Radar: an in-building rf-based user location and tracking system. In INFOCOM 2000. Nineteenth Annual Joint Conference of the IEEE Computer and Communications Societies. Proceedings. IEEE, volume 2, pages $775-784$ vol.2, 2000.

5. P. Bahl, A. Balachandran, and V. Padmanabhan. Enhancements to the radar user location and tracking system. Technical report, Microsoft Research Technical Report, February 2000, 2000.

6. J. J. Astrain, J. Villadangos, J. R. Garitagoitia, J. R. González de Mendívil, and V. Cholvi. Fuzzy location and tracking on wireless networks. In MobiWac '06: Proceedings of the 4th ACM international workshop on Mobility management and wireless access, pages 84-91, New York, NY, USA, 2006. ACM.

7. R. Kumar K., V. Apte, and Y. A. Power. Improving the accuracy of wireless lan based location determination systems using kalman filter and multiple observers. In Wireless Communications and Networking Conference (WCNC), Las Vegas, April 2006, 2006.

8. Wenyao Ho, Asim Smailagic, Daniel P. Siewiorek, and Christos Faloutsos. An adaptive two-phase approach to wifi location sensing. In PERCOMW '06: Proceedings of the 4th annual IEEE international conference on Pervasive Computing and Communications Workshops, page 452, Washington, DC, USA, 2006. IEEE Computer Society.

\footnotetext{
7 http://en.wikipedia.org/wiki/3G
} 
9. G. V. Zàruba, M. Huber, F. A. Kamangar, and I. Chlamtac. Indoor location tracking using rssi readings from a single wi-fi access point. Wirel. Netw., 13(2):221-235, 2007.

10. Mathieu Hopmann, Daniel Thalmann, and Frederic Vexo. Thanks to geolocalized remote control: The sound will follow. In $C W$ '08: Proceedings of the 2008 International Conference on Cyberworlds, pages 371-376, Washington, DC, USA, 2008. IEEE Computer Society.

11. Sanjeev Dhawan. Analogy of promising wireless technologies on different frequencies: Bluetooth, wifi, and wimax. In AUSWIRELESS '0\%: Proceedings of the The 2nd International Conference on Wireless Broadband and Ultra Wideband Communications, page 14, Washington, DC, USA, 2007. IEEE Computer Society.

12. David Patrick and Robert Morrow. WiFi and Bluetooth Coexistence. McGraw-Hill Professional, 2004.

13. Roberto Member-Corvaja. Qos analysis in overlay bluetooth-wifi networks with profile-based vertical handover. IEEE Transactions on Mobile Computing, 5(12):1679-1690, 2006.

14. R. McSweeney. Sumi - a psychometric approach to software evaluation. Unpublished MA (Qual) thesis in Applied Psychology, University College Cork, Ireland, 1992.

15. Susannah Ravden and Graham Johnson. Evaluating usability of human-computer interfaces: a practical method. Halsted Press, New York, NY, USA, 1989.

16. Carol Stoak Saunders and Jack William Jones. Measuring performance of the information systems function. J. Manage. Inf. Syst., 8(4):63-82, 1992.

17. G. Wong and R. Rengger. The validity of questionnaires designed to measure usersatisfaction of computer systems. Technical report, National Physical Laboratory report DITC 169/90, Teddington, Middx., UK, 1990. 